\title{
Los eventos privados: del conductismo metodológico al interconductismo
}

\author{
Private events: from methodological \\ behaviorism to interbehaviorism
}

\begin{tabular}{l|l|l} 
Recibido: marzo 18 de 2010 ～Revisado: abril 23 de 2010 Aceptado: agosto 8 de 2010
\end{tabular}

\author{
ROBERTO BUENO** \\ Universidad de San Martín de Porres, Lima, Perú \\ Universidad Nacional Federico Villarreal, Lima, Perú
}

SICI: 1657-9267(201112)10:3<949:EPCMAI>2.3.TX;2-Y

Para citar este artículo. Bueno, R. (2011). Los eventos privados: del conductismo metodológico al interconductismo. Universitas Psychologica, 10 (3), 949-962.

* Ensayo teórico.

** Escuela Profesional de Psicología. Av. Tomás Marsano 242, Lima, Perú. E-mails: bueno@psicologia. usmp.edu.pe; robuenoc@hotmail.com

\section{RES UMEN}

Como supuestos eventos solo accesibles a un observador, los llamados eventos privados plantean un reto importante a la Psicología Conductista. El problema de los eventos privados fue abordado desde las tres variantes históricas del conductismo: el conductismo metodológico, el conductismo radical y el interconductismo. El argumento básico de las dos últimas perspectivas, y que las diferencia de la primera, es que los eventos privados están comprendidos en el marco de una ciencia objetiva de la conducta. El análisis de estas distintas posturas permite comprobar el gradual avance de la teoría de la conducta, en el tratamiento de este tema, hacia una visión enteramente monista.

Palabras clave autor

Conciencia, conductismo, eventos privados, interconductismo, introspección, subjetividad.

Palabras clave descriptores

Historia de la psicología, epistemología, Skinner, Kantor, Ribes.

\section{A B S T R A C T}

As supposed events being accessible to a unique observer, private events posit an interesting challenge to a behavioristic psychology. Three historical variants of behaviorism dealt with the problem of private events: methodological behaviorism, radical behaviorism and interbehaviorism. The basic argument of the two last views, and that distinguishes them from the former, is that private events are comprised into the scope of an objective science of behavior. The analysis of these accounts permits us to confirm a gradual advance in behavior theory, in dealing with this issue, toward a totally monistic view.

Key words author

Behaviorism, consciousness, interbehaviorism, introspection, private events, subjectivity.

Key words plus

History of psychology, epistemology, Skinner, Kantor, Ribes. 


\section{Introducción}

El término evento privado se adoptó en la teoría de la conducta para hacer referencia a ciertos supuestos eventos que ocurren en un individuo dado y que solo él puede observar, o a los que solo dicho individuo puede tener acceso. En la medida en que parecen constituir una realidad innegable, pero no sujeta a la inspección pública, siempre han planteado un reto interesante a la Psicología Conductista. En lo que sigue, se tratará de mostrar las visiones que acerca del problema de los eventos privados y, con menor énfasis, la conciencia, han mantenido el conductismo metodológico, el conductismo radical y una variante contemporánea del interconductismo. El objetivo de este análisis es poner de relieve la gradual desmitificación del tema de los eventos privados, mostrando que, al abocarse a él, la Psicología Conductista ha avanzado desde una postura todavía compatible con una visión dualista hacia una estrictamente monista y, por lo tanto, plenamente concordante con sus fundamentos filosóficos.

\section{Eventos encubiertos y eventos privados}

Los eventos son ocurrencias en entidades concretas (Clavijo, 2007). Un hecho corresponde al estado en que pueden encontrarse determinadas cosas (hecho estático), o a un cambio en dicho estado (hecho cinético). Por tanto, un evento es una subclase de hechos cinéticos - eventos son los hechos cinéticos que transcurren rápidamente-, y los eventos, como cualesquier hechos, siempre transcurren en las cosas concretas (Bunge, 2006, p. 16). En la teoría de la conducta, los eventos privados suelen ser descritos como hechos "dentro" del individuo que no pueden conocerse social o públicamente, a menos que el propio individuo los reporte. Donahoe y Palmer (1994), por ejemplo, afirman que los eventos privados son "el mundo interno del individuo, por ejemplo, los estímulos que se originan bajo la piel, a los que cada persona tiene un acceso único" (p. 361). Hablando sobre la conducta verbal, Catania (1998) señala que los eventos privados son "en la conducta verbal, los eventos accesibles sólo al hablante (usualmente, eventos debajo de la piel)" (p. 403). El término "encubierto", por otro lado, se refiere a eventos que, bajo ciertas condiciones, son poco o difícilmente accesibles, pero de los que no se predica que sean intrínsecamente privados, en el sentido ya mencionado. Si las condiciones de observación mejoran, usualmente mediante instrumentos, y puede entonces accederse a dicho evento, este deja de ser "encubierto". Suele aplicarse el término 'encubierto' en relación con la actividad de los órganos y sistemas internos. Watson (1919, p. 14; 1924 , p. 15) había descrito a tal actividad como respuestas internas o implícitas. Sin embargo, se trataba de eventos no intrínsecamente inobservables: "Las respuestas internas o implícitas son difíciles de observar, no a causa de que sean inherentemente distintas de las respuestas externas o abiertas, sino simplemente porque están escondidas a los ojos" (Watson, 1924, p. 15).

El siguiente ejemplo subraya la diferencia entre lo encubierto y lo privado. Las respuestas autonómicas características de una reacción emocional son detectables, algunas a simple vista (abiertas) y otras solo con ayuda de instrumentos (encubiertas). En cambio, la experiencia emocional, que supuestamente se expresa a través de esas respuestas, se considera privada, en el sentido de ser completamente inaccesible a otros, ya sea a simple vista o con instrumentos. De ahí que, como lo indica Alcaraz (1982),

(...) el estudio de las reacciones encubiertas o el de la actividad cerebral concomitante, no resuelve el problema de la investigación sobre la subjetividad (...) el registro de una respuesta encubierta nos dice poco acerca de lo que se tiene como experiencia subjetiva. (p. 36)

Aunque en ocasiones las respuestas encubiertas han sido tratadas como componentes de los eventos privados (por ejemplo, Tourinho, 2006), debe decirse que las respuestas mismas, aunque no sean aparentes a un segundo observador, nunca son privadas. El hablar inaudible es una respuesta encubierta, pero siempre pública y verificable, como 
evento que puede ser registrado sensorialmente de manera directa o a través de instrumentos, en la medida que es un evento físico. Sin embargo, cuando se afirma que respuestas de esa clase son eventos privados, quizá se quiere decir que lo privado no son las respuestas mismas, sino su contenido o significado, esto es, lo que se dice, lo que se ve o lo que se siente, es decir, la experiencia que se dice que acompaña a la actividad organísmica. El evento privado está constituido, entonces, según la concepción usual, por todo contenido o experiencia solo accesible al individuo y que acompaña, o incluso conduce a, la respuesta, encubierta o abierta.

\section{De la Psicología introspectiva al Conductismo metodológico}

De alguna manera, la adopción del método introspectivo era la respuesta comprensible en el marco del primer intento por introducir métodos científicos en Psicología. El método elegido, obviamente, respondía a las características supuestas del objeto de estudio. "La Psicología es la Ciencia de la Vida Mental, tanto en sus fenómenos como en sus condiciones. Los fenómenos son cosas tales como las que llamamos sentimientos, deseos, cogniciones, razonamientos, decisiones, etc.", escribió William James (1890, p. 1). Si la física debe, metodológicamente, observar las cosas que están a nuestro alrededor, la Psicología, rama científica de similar status, debe emplear el mismo método, solo que su objeto de estudio no son esas cosas externas, sino las internas: "para el psicólogo, entonces, las mentes que él estudia son objetos [cursivas en el original] en un mundo de otros objetos" (James, 1890, p. 183). Los contenidos accesibles a la observación se denominaron colectivamente la conciencia. La introspección se consideró entonces un acceso directo a estos contenidos: "introspección significa (...) mirar dentro de nuestras propias mentes y reportar lo que descubrimos allî" (James, 1890, p. 185). E. B. Titchener, el reconocido introspeccionista, estaba convencido de que la introspección era una variedad de observación científica, en nada esencialmente distinta de la observación practicada en otras disciplinas: "iHay un método psicológico especial, una peculiar manera de trabajar que (el psicólogo) debe adoptar en su estudio de los fenómenos mentales? La respuesta es: No. Su método es el de la ciencia en general" (Titchener, 1915, p. 19). Por tanto,

(...) el método de la psicología (...) es la observación. Para distinguirla de la observación de la ciencia física, la cual es inspección, un mirar a, la observación psicológica ha sido llamada introspección, un mirar dentro. Pero esta diferencia de nombre no debe cegarnos con respecto a la semejanza esencial de los métodos. (Titchener, 1928, p. 20)

Pero, como observó Leahey (1992), varios autores norteamericanos expresaron desde comienzos del siglo XX muchas dudas e inconformidades respecto de la Psicología introspectiva. De algún modo, el pronunciamiento de Watson (1913) vino a formalizar, y a dar un nombre, a esas diversas expresiones. Fueron varios los argumentos de Watson (1913) en contra de la introspección, como método psicológico, y de la conciencia, campo explorado mediante dicho método, como objeto de conocimiento de la Psicología. Respecto de la introspección, señaló lo impreciso de su lenguaje, atribuyendo a esa deficiencia los frecuentes desacuerdos entre los observadores. Otra de sus objeciones enfatizaba el hecho de que ninguna ciencia practica la introspección. Y en cuanto a la conciencia, fueron dos los principales reparos. En primer lugar, la interpretación de un resultado experimental no requiere en absoluto describir -ni por observación directa, ni por inferencia- los contenidos conscientes del sujeto. El individuo responde de manera precisa a las condiciones experimentales, de modo que describir una estación intermedia entre esas condiciones y la conducta resulta del todo innecesario. En segundo lugar, Watson (1924) también indicó que el estudio de la conciencia correspondía a objetivos de conocimiento propios de la tradición filosófica dualista, como, por ejemplo, el problema de las dos sustancias, mente y cuerpo, objetivos que, según él, transcendían las metas del conocimiento científico. Para Watson, la conciencia solo era un instrumento para hacer la observación de 
las cosas y no un objeto para ser observado. Como él mismo lo expresó:

[Se] puede hacer omisión de la conciencia en un sentido psicológico. A partir de esta premisa, concluimos que la observación de "estados de conciencia” es tan poca tarea del psicólogo como lo es del físico. Podemos llamar a esta posición el retorno al empleo no reflexivo e ingenuo de la conciencia. En este sentido, se puede decir que la conciencia es el instrumento o herramienta con la cual trabajan todos los científicos. (Watson, 1913, p. 176; el mismo texto es repetido en Watson, 1914, p. 27)

La cita presenta el postulado fundamental de lo que después Skinner (1945) llamó conductismo metodológico. Según Hayes, Wilson y Gifford (1999), la postura de Watson respecto, específicamente, de los datos fundamentales de la Psicología (al margen de otros aspectos de su trabajo), y al menos como se expresa en el pasaje citado, constituyó una forma de conductismo metodológico, pues, para Watson, "aun si las actividades mentales u otros no movimientos existieran, ellos no podrían constituir el objeto de la psicología científica porque era imposible conseguir un acuerdo público respecto de su ocurrencia” (p. 153). En suma, el conductismo metodológico consiste en aceptar la existencia de eventos privados y, a la vez, en su exclusión de toda consideración científica, por no ser públicamente observables. En esencia, esta misma caracterización del conductismo metodológico es compartida por autores como Zuriff y Staddon (citados por Uttal, 2000, pp. 23-24).

Un escrito representativo de esta posición es el artículo clásico y muy citado de Spence (1948). De acuerdo con Spence, el conductista asume una posición según la cual los datos científicos, incluidos los psicológicos, se encuentran en la experiencia inmediata del científico. Afirma, explícitamente, que el psicólogo tiene como tarea imponer orden y significado en los hechos proporcionados por la experiencia inmediata. Spence continúa proponiendo una diferencia entre aquellas experiencias sensoriales derivadas de acontecimientos externos (estímulos externos) al individuo, de aquéllas otras que dependen de eventos (estímulos) internos. Y seguidamente afirma que, al igual que en la física, la Psicología se ocupa solamente de las experiencias de origen externo, procedentes de la conducta "manifiesta" (observable, pública, etc.) de organismos diferentes del observador. Esta posición se justifica señalando que solo las manifestaciones o eventos "externos" son "públicos" y "verificables". Los eventos internos, aun cuando son tan físicos como los externos, resultan ser difícilmente detectables y su reporte por el individuo no es verificable. En consecuencia, si bien no se desecha del todo el material de las experiencias de origen interno, estas se consideran con suma cautela. En suma, el postulado fundamental del conductismo metodológico, entendido de esta manera, es el siguiente: solo los eventos que el observador capta mediante sus exteroceptores constituyen datos científicos, pues solo estos se prestan para lo que sus partidarios estipularon como el requisito básico del método científico: la observabilidad pública.

Spence (1948) afirma que estas tesis fueron avaladas, o eran compartidas, por positivistas lógicos como Carnap y Feigl y por el operacionismo de Bridgman. En realidad, fue la posición esencialmente asumida por psicólogos como Edwin Boring y S.S. Stevens. El mismo Spence, según Smith (1986, p. 19), estuvo "afiliado a una forma modificada de positivismo lógico y veía a éste como una orientación metodológica apropiada para la psicología”. Pero tal postura, apoyada por (o en) el positivismo lógico y el operacionismo, nunca dejó de ser, en los hechos, dualista. Primero, la experiencia continuó siendo parte de un mundo de subjetividad, accesible solo mediante introspección, y en consecuencia, privada. En otras palabras, el conductismo metodológico aceptó la concepción dualista tradicional, según la cual existe un mundo físico públicamente observable que constituye el objeto de conocimiento de la ciencia, y otro esencialmente privado, ajeno a la observación científica, pero en el que, entre otros fenómenos, existen las sensaciones en las que se refleja el mundo público. Y segundo, porque la práctica de definir operacionalmente los términos que hacen referencia a la subjetividad (y los términos mentalistas en 
general), solo perpetuaba el uso de tales términos, como descripciones de eventos supuestamente reales pero ocultos. Ambos resultados fueron criticados por Skinner (1945). Para Skinner, el mundo de la experiencia no simplemente debe ser asumido, a la manera de un instrumento mediante el cual se practican las observaciones, y en donde habitan los datos, sino que ese mismo mundo es también parte de la conducta que hay que explicar. Y además, el problema de los términos mentalistas no es el de formular para ellos definiciones objetivas, es decir, operacionales, como si tuvieran un referente real. El problema de tales términos radica, más bien, en hallar las condiciones objetivas en las cuales se usan o, dicho en el lenguaje contemporáneo, tratar el uso de tales términos como respuestas verbales y determinar las contingencias de reforzamiento de las que dichas respuestas verbales forman parte.

Para finalizar esta sección, es importante también recordar que con la excepción de Spence y de algunos otros, pocos de los más importantes conductistas cuya carrera profesional se desarrolló en plena efervescencia positivista lógica, se vieron particularmente influidos por alguna variante de dicha filosofía (véanse, por ejemplo, Hatfield, 2002; Smith, 1986). Skinner, en particular a partir de 1945, mantuvo puntos de vista encontrados frente a las concepciones del positivismo lógico y del operacionismo (véase, por ejemplo, Moore, 1985). Por otro lado, muchos de aquellos conductistas consideraron seriamente la necesidad de abordar el problema de la conciencia, como fue el caso, por ejemplo, de Lashley (1923) o de Tolman (1927), y obviamente, de Skinner. El objetivo, por supuesto, no era hacer un estudio introspectivo de la conciencia, sino llegar a explicar los fenómenos conscientes en el marco de una teoría general de la conducta.

\section{La posición del conductismo radical}

Los eventos privados, según Skinner, constituyen el mundo debajo de la piel, el universo psicológico interno, del que una ciencia de la conducta debe necesariamente ocuparse:
Una adecuada ciencia de la conducta debe considerar los eventos que tienen lugar debajo de la piel del organismo, no como mediadores fisiológicos de la conducta, sino como partes de la propia conducta. Esta ciencia puede tratar con dichos eventos sin asumir que ellos tienen alguna naturaleza especial o que deben ser conocidos de alguna manera especial. La piel no es tan importante como un límite. Los eventos privados y los eventos públicos tienen las mismas clases de dimensiones físicas. (Skinner, 1963, p. 953)

Por tanto, los eventos privados no deben constituir problema alguno para una ciencia de la conducta. Dos son las ideas fundamentales. Primero, los denominados eventos privados son realmente eventos concretos que ocurren dentro del individuo, pero son de naturaleza física. Segundo, dichos eventos pueden ser observados por el individuo en quien ocurren, pero la posibilidad de realizar dicha observación se concreta por medio de procesos (de aprendizaje) públicos y observables. Estas ideas fundamentales fueron expuestas repetidamente por Skinner (p. ej., 1945, 1953, 1963, 1974) y pueden explicarse con más amplitud del siguiente modo.

Skinner comienza sosteniendo que "una pequeña parte del universo está contenida dentro de la piel de cada uno de nosotros. No hay razón por la que esa parte debería tener un status físico especial a causa de que exista dentro de estos límites" (Skinner, 1974, p. 21). Esa pequeña parte de universo es la estimulación propioceptiva e interoceptiva, la estimulación proveniente de la propia actividad del organismo. Dicha estimulación es esencialmente privada, en el sentido de que no puede afectar a nadie más excepto al individuo que la produce. Por tal razón, Skinner señala que "la respuesta del individuo a un diente inflamado (...) no es como la respuesta que cualquiera otro puede dar a ese diente en particular, debido a que nadie más puede establecer la misma clase [cursivas añadidas] de contacto con él" (Skinner, 1953, p. 257). El problema de la privacía, entonces, se plantea únicamente porque existe cierta clase de estimulación interna a 
la que solo su poseedor puede acceder. Pero dichos estímulos privados son, en algunos casos, generados por respuestas encubiertas, incluso verbales. Así, el mundo privado está hecho de estímulos generados por la actividad verbal o no verbal, innata o aprendida, del organismo.

Para Skinner, la adquisición del autoconocimiento se da como un proceso conductual y, en consecuencia, es susceptible de observación y análisis. En lo fundamental, describió tal proceso como un aprendizaje de respuestas, verbales entre ellas, controladas discriminativamente por la estimulación interna. El observar o describir eventos privados significa, entonces, emitir respuestas, verbales o de otro tipo, a la estimulación interna. En suma, básicamente, no existe una diferencia fundamental entre describir eventos externos y describir eventos internos, pues en los dos casos, se trata de respuestas discriminadas a objetos y eventos concretos. Sin embargo, el proceso de entrenamiento de la descripción de eventos privados tropieza con ciertas dificultades, dado que el instructor no tiene acceso a los estímulos privados que deberán adquirir una función discriminativa respecto de las respuestas verbales que está enseñando, lo que no le permite reforzar las respuestas apropiadas del aprendiz, es decir, las respuestas verbales correctas a los estímulos privados del aprendiz. Estas dificultades se superan parcialmente con base en que muchos estímulos privados están correlacionados con diferentes clases de eventos públicos. Se puede resumir lo que podemos llamar los cuatro mecanismos mediante los cuales se aprende el autoconocimiento. En primer lugar, el reforzamiento se puede administrar con base en estímulos públicos que acompañan regularmente a estímulos privados. En segundo lugar, se puede administrar con base en respuestas que suelen acompañar a los estímulos privados. En tercer lugar, el reforzamiento puede procurarse a respuestas que describen la conducta abierta del hablante. En este caso, en virtud de que la conducta tiene componentes también encubiertos (la estimulación propioceptiva generada por la actividad del sujeto), la misma descripción reforzada queda asociada a esos componentes encubiertos, pudiendo ser evocada cuando la conducta se rea- liza a nivel encubierto. Incluso, la misma respuesta verbal puede volverse encubierta. Y en cuarto lugar, una respuesta verbal adquirida en relación con ciertos estímulos públicos, puede emitirse también en presencia de estímulos privados similares o que comparten ciertas propiedades con los estímulos públicos. De esta manera, el análisis de Skinner "es, con mucho, un análisis de cómo una comunidad verbal podría enseñar a sus miembros a usar términos subjetivos para describir eventos privados (e.g. un dolor de muelas)" (Schneider \& Morris, 1987, p. 34).

Sin embargo, observa Skinner, nunca será perfecta la correlación entre los eventos públicos en los que se basa el reforzamiento de las respuestas verbales y los eventos privados a los que esas respuestas deben quedar asociadas. Es por esto, y por el hecho de que no se dispone de todos los receptores capaces de captar todos los estímulos privados, que el autoconocimiento jamás será más exacto que el conocimiento de los eventos externos (Skinner, 1974). Con todo, el análisis de Skinner muestra que el autoconocimiento es un producto social: "Ser consciente, como una forma de reaccionar a la propia conducta de uno mismo, es un producto social (...) sólo porque la conducta es importante para la sociedad, la sociedad a su vez la hace importante para el individuo" (Skinner, 1945, p. 277).

Friman, Wilson y Hayes (1998) citan un comentario de Zuriff, quien había señalado que "no hay evidencia actualmente disponible de que (...) estos eventos privados son estímulos en el sentido de conformarse a las mismas leyes que sus contrapartes abiertas" (Zuriff, 1988 citado por Friman et al., p. 707). La respuesta de Friman et al., consistió en citar evidencia de que los eventos privados pueden adquirir propiedades discriminativas o constituir relaciones de equivalencia entre sí, es decir, que los eventos privados funcionan como cualquier otro estímulo. Sin embargo, los eventos privados estudiados en los experimentos citados eran realmente estímulos privados (es decir, según hemos visto ya, estímulos propioceptivos o interoceptivos). Algunos de esos experimentos concernían a la discriminación de drogas o discriminación de los 
efectos subjetivos de las drogas. En casos así, se puede mostrar que esos efectos (cambios somáticos provocados por la sustancia) actúan como estímulos capaces de controlar respuestas abiertas. Branch (2006, pp. 411-413) comentó que, al margen de su interés intrínseco, estos estudios tienen implicaciones para investigar empíricamente el control de la conducta por eventos privados. Sin embargo, la respuesta de Friman et al. y los comentarios de Branch dan por sentado precisamente lo que Zuriff cuestiona: que los eventos privados realmente sean solamente los estímulos privados. Desde una visión skinneriana, la única posible respuesta a Zuriff era, más bien, que los eventos privados (sentimientos, sensaciones, etc.) se igualan a los estímulos privados (o sea, estímulos de naturaleza interoceptiva o propioceptiva) básicamente por razones filosóficas, ontológicas. En realidad, es posible que ninguna evidencia -al menos por el momento- pueda probar que tal identidad es verdadera.

En sus trabajos más recientes, Skinner fue poniendo énfasis en la idea que se puede resumir así: "lo que sentimos son condiciones de nuestro cuerpo, la mayor parte de las cuales están estrechamente relacionadas con la conducta y con las circunstancias en las que nos comportamos" (Skinner, 1975, p. 43; véase también Skinner, 1974). Esas declaraciones implican que no se puede diferenciar diversos contenidos de lo que se siente solamente sobre la base de la topografía de los eventos físicos involucrados. En otras palabras, puede haber distintas respuestas verbales frente a un mismo estímulo privado. Este punto debe considerarse en relación con la definición de una operante como respuesta funcional. Lo que define a una operante no es solamente (ni básicamente) su topografía, sino las contingencias de las que forma parte (Skinner, 1969, cap. 5). La misma respuesta puede tener funciones diferentes, es decir, constituir operantes diferentes, en función de las contingencias específicas en las que está integrada (y por tal razón, no es lo mismo respuesta que conducta. La respuesta es simplemente un evento físico, en tanto que la conducta, si cabe la siguiente simplificación, es tal evento físico en relación funcional con otros eventos, externos al sujeto. Por tanto, si hablamos de respuestas verbales a los componentes encubiertos de la propia conducta, lo que se siente no dependería tan solamente de la forma física de tal conducta (o respuesta), sino, básicamente, de su función, esto es, de las contingencias. Es decir, cuando el sujeto aprende a describir eventos privados, no aprende solamente a responder con esta o aquella palabra específicas a determinadas condiciones corporales específicas, sino que el término empleado puede quedar también asociado a las circunstancias en que ocurre la conducta asociada a tales eventos y las contingencias de la que forma parte. Es de presumir, por ejemplo, que el empleo de términos como culpa, miedo o remordimiento queda asociado al mismo tipo de eventos encubiertos, pero el uso de uno u otro de esos términos dependerá también de las circunstancias específicas en que surge el estímulo privado, entre las cuales se encuentra el agente que administra el castigo. En el análisis original de Skinner (1945) no se preveía esta nueva posibilidad, la cual acentúa más bien el papel de las contingencias situacionales, por encima de las propiedades físicas de los estímulos privados, en la determinación de lo que se ve o se siente introspectivamente en la situación.

Dos objeciones pueden oponerse a la visión del conductismo radical sobre los eventos privados. Primero, tal análisis mantiene la existencia de eventos que son físicos (y por tanto, existentes con anterioridad a toda observación y/o descripción por parte del individuo) y, sin embargo, solo accesibles a un observador. Como se señala en el pasaje ya citado de Alcaraz (1982), el registro instrumental de tales eventos no resuelve el problema, no solo porque el instrumento o su actividad distorsionan el evento registrado, sino, principalmente, porque el instrumento solo proporciona lecturas que para el observador constituyen estímulos exteroceptivos. La única forma de hacer realmente accesible a un segundo individuo un evento privado producido en otro, es que exista alguna conexión entre los sistemas nerviosos de ambos. Kantor (Pseudonym: Observer, 1981) y Ribes (1982) señalaron que hablar de eventos concretos pero invisibles a la observación pública, contiene todavía un residuo de dualismo. En segundo lugar, muchos términos 
que describen presuntos eventos privados, como pensar o ver, y que parecen referirse a actividades encubiertas, bien podrían ser simples reificaciones del lenguaje ordinario (Ryle, 1949). Muchos conductistas usan liberalmente tales términos, como descripciones de eventos privados. Como Ribes (2004) señala, parece que no han advertido que, por ejemplo, el término pensar no se refiere a una actividad concreta, sino a muchas y muy variadas actividades en las que hay respuestas implicadas, tanto abiertas como encubiertas, así como a las circunstancias en que tales actividades se realizan. En tal caso, la búsqueda de respuestas encubiertas que correspondan a cosas como pensar o ver sería una empresa vana.

\section{Un análisis interconductual: los eventos privados como instancias de eventos sustitutivos}

Como un desarrollo a partir del metasistema propuesto por Kantor (1924-1926, 1958), Ribes y López (1985) diseñaron una taxonomía de la conducta en la que cobra una importancia trascendental la distinción entre los eventos psicológicos presustitutivos y los sustitutivos, distinción que es fundamental para situar adecuadamente el problema de los eventos privados. Los individuos pueden interactuar con los objetos y eventos bajo contingencias que dependen de las propiedades físicas o de las propiedades ecológicas o de las propiedades convencionales de dichos objetos y eventos. La conducta operante es un caso de interacción con las propiedades físicas de los objetos. En tal tipo de conducta, la relación contingencial se da entre eventos de estímulo y de respuesta presentes físicamente y, además, la existencia de tales relaciones se posibilita estrictamente por las características físicas de los eventos relacionados. Por ejemplo, la relación entre el acto de clavar un clavo (respuesta) y el efecto de introducirlo (estímulo) es posible solo en la medida en que la respuesta adopte una morfología determinada, adecuada a dicho cambio de estímulo particular. En una relación operante no se da la relación funcional entre una respuesta y, por ejemplo, un cambio de estímulo distante en el tiempo. Al clavar, por ejemplo, el efecto de la respuesta es inmediato, está presente en la situación.

En la sustitución referencial la función de la respuesta no es provocar un efecto físico inmediato, sino una más compleja, la de introducir nuevas contingencias en la situación para otro individuo o para uno mismo. Las contingencias introducidas, además, no existen en virtud de las características físicas de los eventos así relacionados, sino que han sido construidas socialmente, son convencionales. Esta posibilidad de introducir contingencias en la situación implica, entonces, que el sujeto responde a eventos o propiedades no presentes físicamente en la situación. Estas propiedades de un objeto no presentes físicamente en ese objeto, existen en la medida en que son atribuidas socialmente a él y constituyen, por tanto, las propiedades convencionales de dicho objeto-estímulo. Por propiedades convencionales, o socialmente atribuidas, del objeto-estímulo debe entenderse las relaciones funcionales convencionalmente establecidas entre dicho objeto o situación y determinadas formas de respuesta. En las interacciones sustitutivas, la respuesta mediadora debe también, necesariamente, poseer una morfología convencional, es decir, arbitraria respecto de las propiedades físicas de los eventos a los que se responde. Como veíamos, la morfología del acto de clavar no puede ser arbitraria respecto del efecto requerido en el objeto-estímulo. Sin embargo, si la respuesta no es a propiedades físicas sino convencionales del objeto-estímulo, dicha respuesta no requiere necesariamente de una determinada morfología, que se corresponda físicamente con los cambios de estímulo relacionados con aquélla. En suma, las interacciones sustitutivas referenciales suponen el responder al objeto-estímulo, en términos de las prácticas culturales que estipulan la reacción apropiada a dicho objeto-estímulo, a su vez en función de un contexto o circunstancias sociales determinadas.

La interacción sustitutiva referencial se da como un proceso de mediación de las acciones de otro, o de uno mismo, respecto de una situación u objeto particular, al que se llama 'referente'. Pero es una forma de mediación en la que tanto el me- 
diador, o referidor, y a continuación el mediado, o referido, interactúan no con las propiedades físicas del referente, en el marco de contingencias que existen en virtud únicamente de dichas propiedades, sino que interactúan con sus propiedades convencionales, lo que equivale a decir, que interactúan con el objeto en el marco de contingencias construidas socialmente. El término sustitución tiene precisamente el sentido de 'remplazo'. En la sustitución referencial el mediador remplaza, para el mediado, las contingencias a través de las cuales este último interactúa con el referente. Sin embargo, esta función de sustituir contingencias para el mediado, solo puede cumplirse si el mediador es también, previamente, capaz de interactuar convencionalmente con el referente. Esta última aclaración es pertinente respecto de la crítica a la definición de conducta verbal propuesta por Skinner (1957), quien la señalaba como la conducta cuyo reforzamiento es mediado por otro individuo. De este modo, siguiendo un ejemplo del propio autor, la presión de la palanca por parte de la rata sería una forma de conducta verbal (un mando), en la medida que el reforzamiento de tal respuesta es mediado por el experimentador. Sin embargo, la presión de palanca por la rata no sustituye contingencias para el experimentador. En la sustitución referencial ambos, mediador y mediado, se comportan sustitutivamente respecto del objeto-estímulo.

Como se dijo, la convencionalidad (arbitrariedad) de las propiedades de los eventos de estímulo y de respuesta propios de las interacciones sustitutivas, permite que la respuesta pueda realizarse independientemente de las características físicas del objeto al que se responde. E incluso, el objeto puede hallarse físicamente ausente, lo que configura aquello que Kantor (1924) llamaba una respuesta implícita (obsérvese que aquí, implícito se usa en un sentido muy distinto de como lo empleaba Watson, 1919, 1924). Por otro lado, como indican Ribes y López (1985), el simple uso de una respuesta con morfología convencional (por ejemplo, la emisión del sonido de una palabra) no implica necesariamente sustitución de contingencias. Una respuesta con morfología convencional, pero sin función sustitutiva, sería la de decir ihola!, cuando tal respuesta solo tiene un efecto físico inmediato. Asimismo, una respuesta a un estímulo con morfología convencional (un estímulo producido por la respuesta convencional de otro), pero no teniendo tal respuesta una función sustitutiva, sería la de una paloma que pique un disco en respuesta a la palabra impresa picar. La paloma está respondiendo únicamente a la pauta física de la palabra impresa. La lectura sustitutiva, en cambio, implica entrar en contacto con aquellos eventos o propiedades, no físicamente presentes, de los que trata el texto.

Con estas bases es posible emprender la tarea de re-conceptualizar al menos una categoría particular de lo que usualmente se llama eventos privados: aquellos que consisten en la valoración particular que cada individuo da a sus propias reacciones cuando se comporta en situaciones definidas culturalmente. En esta categoría se incluye preferentemente la mayor parte de lo que en el lenguaje ordinario llamamos sentimientos, sensaciones, dolor y cosas por el estilo. El punto de partida de este análisis es la constatación de que pueden existir, de hecho existen, algunos eventos originalmente no aparentes, o no visibles, incluso para la persona en quien ocurren, aunque el problema de los eventos privados no es en realidad un problema de eventos no aparentes que el individuo reporta. Como observó Ribes (1982):

Dada la existencia de eventos no aparentes, incluso para la misma persona que los produce mediante su reactividad orgánica, el problema se formula en términos de la génesis de las descripciones sociales que permiten al individuo responder a dichos eventos físicos en la forma de eventos referenciables como "acontecimientos privados" o "eventos internos" (...) el problema se desplaza de la suposición de que existe en efecto un mundo privado interno, que adquiere funcionalidad hacia el exterior por medio de las autodescripciones que el individuo realiza vía el aprendizaje de cómo referirse a ese mundo, hacia el análisis de cómo la comunidad lingüística, mediante las convenciones descriptivas, crea o construye en el individuo que alcanza un nivel particular de evolución conductual, formas reactivas que le permiten referirse a interacciones con otros y con su propia 
reactividad (antes no aparente incluso a él como eventos), en términos propios que corresponden a la funcionalidad pública y social de dichas interacciones. (pp. 19-20)

La cita precedente prefigura el abordaje que propone Ribes para el problema. Su análisis puede plantearse de la siguiente manera.

En primer lugar, y en contra de lo sostenido por Skinner, en principio existe en el individuo tan solo reactividad biológica. A su vez, dicha reactividad carece, como expresa Ribes, de funcionalidad psicológica, lo que define a tal evento como un no estímulo, un evento sin función de estímulo. En consecuencia, según Ribes, es inexacto afirmar que la conducta de describir eventos privados, consiste en respuestas verbales discriminadas a estímulos internos. En segundo lugar, la función de estímulo de los eventos internos de respuesta es, según la expresión de Ribes (1982), socialmente construida:

(...) no todo evento o relación física aparente o no aparente posee necesariamente funciones psicológicas y (...) los eventos o relaciones no aparentes que adquieren dicha función sólo pueden hacerlo a través de la reactividad lingüística que las "construye” como tales (...) la identificación de la propia reactividad, y su posterior influencia como estímulo, sólo es factible en la medida en que se trasciende dicha reactividad directa lingüísticamente, y es la interacción implicada en la respuesta lingüística la que vuelve aparentes y explicitos a los eventos que, como componentes de una circunstancia físicoquímica y biológica, son no aparentes e implícitos a otro observador (...). (p. 23)

En estos y otros pasajes, Ribes utiliza los términos lenguaje y lingüístico para referirse a aspectos de las interacciones sustitutivas. Ahora bien, la reactividad biológica propia podría tener, en algunos casos, funciones estimulativas, induciendo ciertas respuestas a sus propiedades físicas únicamente. Por ejemplo, las correcciones posturales en respuesta a ciertos estímulos propioceptivos, a su vez generados por cambios en la propia postura, etc. Tales casos no corresponderían propiamente a lo que estamos analizando como eventos privados. Ribes insiste en que en los auténticos eventos privados, la función estimulativa de la propia reactividad, es generada socialmente. Es claro que, en tal caso, estamos hablando de una función estimulativa no simplemente física sino convencional. Este proceso de hacer aparente la propia reactividad para el individuo no se da en cuanto el niño comienza a hablar, pues en esa etapa de desarrollo sus respuestas morfológicamente convencionales no tienen necesariamente una función sustitutiva. Es necesario que el niño comience a interactuar sustitutivamente con las cosas, pero el proceso no se detiene ahí, ya que los eventos privados aparecen en cuanto el individuo se convierte él mismo en referente. El evento privado es, entonces, la relación sustitutiva que el individuo establece con su propia reactividad, la que consiste en una respuesta convencional a las propiedades convencionales de su propia reactividad, y en cuanto tal reactividad (sea motora, verbal o de los órganos internos) participa en una interacción con otros bajo la regulación de normas culturales. Como lo resume Ribes (1982):

El acto lingüístico de referir descriptivo de eventos "privados" no corresponde a una "identificación" de eventos concretos que tienen la propiedad de "privacía" o "internalidad" (...) sino que constituye, como forma de interacción con la propia reactividad en un contexto social -es decir, frente a la reactividad de otros- el evento privado mismo. (p. 24)

En el episodio llamado evento privado, el individuo responde a los estímulos originados en su propia reactividad de la manera en que socialmente se prescribe una respuesta a dicha clase de estímulos, pero, precisamente, el carácter de la respuesta a la reactividad es, por convención, el apropiado a la conducta de la que forma parte dicha reactividad y a la situación en que la misma ocurre. Es decir que la respuesta a la propia reactividad que se concrete en una circunstancia dada dependerá, por normatividad social, de un contexto proporcionado por la conducta de la que forma parte esa reactividad, la situación en que ocurre la conducta y la conducta 
de los otros, presente y futura, frente al episodio. Está claro que es la normatividad social la que determina la manera en que todos estos eventos se relacionan entre sí. Por ejemplo, esa normatividad dicta la pauta de reacción que otros han de seguir frente a una conducta específica de algún individuo, dada una situación en que tal conducta se produce. Cuando se habla de la valoración o del significado de la propia reactividad, se está haciendo referencia, precisamente, al hecho de que dicha reactividad ocurre en el marco de este contexto. Por eso se da el caso de que la reactividad que forma parte de muchas formas distintas de conducta pueda ser prácticamente la misma y, sin embargo, lo que se experimenta subjetivamente puede variar de un contexto a otro. Por ejemplo, un individuo puede interpretar sus propios latidos cardíacos fuertes y rápidos como miedo o como placer o como emocionalmente neutros, en función del contexto en que se produce tal reactividad, como lo mostró el famoso experimento de Schachter y Singer (1962). Lo que cambia de un caso a otro no son las características físicas de las respuestas somáticas, sino el contexto en el que estas ocurren.

De acuerdo con lo examinado, las respuestas verbales que describen eventos privados, lejos de describir un mundo interno, describen más bien (si cabe sostener que describen algo), eventos observables y públicos: la conducta propia, el contexto social o interpersonal situacional, la normatividad cultural y la historia del individuo a través de la cual dicha normatividad adquiere sus funciones reguladoras (Bueno, 1993). Hablar de la tristeza no es hablar de algo que transcurre en el interior, sino que es hablar acerca de cómo uno interactúa en un contexto social específico, con su propia reactividad y con los otros.

Podemos ilustrar brevemente esta concepción mediante una descripción del sentimiento de vergüenza. Como señalan Thomaes, Stegge y Olthof (2010), la vergüenza "involucra conciencia de la desaprobación ajena” (p. 224). El sentimiento de vergüenza, como muchos otros, está relacionado con el castigo y con sus efectos emocionales. Genéticamente, tiene como más remoto antecedente al aprendizaje de respuestas que anticipan un castigo, por ejemplo, respuestas somáticas condicionadas a estímulos que anticipan un castigo. Pero en tanto el individuo transite aún por niveles pre-sustitutivos de desarrollo, las respuestas somáticas generadas por tales castigos, o amenazas de castigo, no pueden sentirse como vergüenza y, si seguimos a Ribes, es posible que tales respuestas sean en todo o en parte inaparentes incluso para el propio individuo. En la etapa sustitutiva persiste la posibilidad de que las respuestas somáticas asociadas al castigo se activen si la conducta castigada se repite. Es lo que puede suceder si alguien es observado desnudo en una situación valorada socialmente como inapropiada, es decir, una situación del tipo de aquellas que involucraron alguna forma de castigo. Pero, en la etapa sustitutiva, no solo se produce una reacción somática frente a la situación (posibilidad que, como queda dicho, tiene sus orígenes en la infancia del sujeto), sino que ahora tal circunstancia se experimenta como vergüenza. La sustitución de contingencias ocurre en la medida que el sujeto no responde a las propiedades físicas de su propia reactividad -suscitada bajo esas circunstancias-, sino que responde a ella como es socialmente prescrito que debe responderse bajo dichas circunstancias. Es decir, como hemos visto, responde a su propia reactividad de una manera que, por normatividad social, corresponde al contexto en el que tal reactividad surge, el cual incluye la conducta de la que tal reactividad forma parte (como escapar; véanse p. ej., Lindsay-Hartz, De Rivera \& Mascolo, 1995), a la situación en que ocurre la conducta y la respuesta de los otros, presente y futura, a tal conducta dada la situación, que en este caso específico es una de censura y desaprobación. Como han explicado algunos autores, la gente avergonzada es la que se siente expuesta (Smith, Webster, Parrott \& Eyre, 2002).

Por último, cabe recordar que las respuestas con función sustitutiva referencial siempre son respuestas a eventos o situaciones particulares (Ribes \& López, 1985), como, por ejemplo, sentir vergüenza respecto de una situación u objeto específico. Una función psicológica todavía más compleja, la sustitución no referencial, implica, en primera instancia, la posibilidad de interactuar con las respuestas que 
tienen función sustitutiva. Es lo que puede ocurrir, por ejemplo, en algunos casos de solución de problemas. Supongamos un individuo que resuelve el problema A, y a continuación resuelve el problema B. Si el sujeto ha verbalizado las soluciones, pero estas son distintas para cada problema, se puede decir que ha interactuado con ellos de manera sustitutiva referencial, es decir, ha interactuado separadamente con dos situaciones específicas. $\mathrm{Si}$, por el contrario, ha elaborado una regla general (a partir de su previa verbalización de cada uno de los dos problemas), y ha procedido a resolver dichos problemas en virtud de dicha regla, su conducta se ajusta a uno de los niveles más sencillos de la sustitución no referencial. Sin embargo, a diferencia del primer caso en que el individuo tendría que idear una nueva solución para el problema C, probablemente diferente de la empleada en los otros problemas, en el segundo caso el individuo puede continuar aplicando la misma regla en la solución de muchos problemas distintos. De igual manera, es posible que un individuo pueda interactuar con sus propias diversas y distintas experiencias de eventos privados, generando más complejas expresiones de su mundo interior (Ribes, 1982). Las sensaciones experimentadas frente a un evento Ay otras distintas experimentadas frente a un distinto evento B podrían, sin embargo, sintetizarse en una nueva forma de vivencia, del mismo modo en que las descripciones verbales de dos problemas distintos pueden sintetizarse en una única regla general.

La naturaleza esencialmente sustitutiva de los eventos privados, de alguna manera es reconocida en el siguiente pasaje de Hayes et al. (1999):

A medida que los humanos aprenden a discriminar y hablar acerca de eventos privados, la ocurrencia de eventos privados trae un conjunto separado de contingencias a la situación [cursivas en el original] (...) Por ejemplo, supongamos que una persona recibe un shock eléctrico en una cámara experimental. La persona, como la rata, se sobresaltará y se sentirá ansiosa. Pero cuando la persona se siente "ansiosa" y es consciente de tal sentimiento, las muchas reglas aprendidas sobre la ansiedad (es mala, significa que eres débil, debe ser resistida, podría dañar mi salud) son incorporadas a la situación (...) lo que sucede a continuación en el mundo de la conducta abierta no es sólo el resultado del shock o de su historia con el shock. Es un resultado de todo ello, más todas las funciones de estímulo derivadas y las contingencias sociales/verbales comprometidas por el hablar de la persona. (pp. 182-183)

En suma, los eventos privados son casos particulares de las interacciones sustitutivas. En consecuencia, no representan ningún problema para una teoría objetiva de la conducta, ni, en última instancia, para el Conductismo como marco filosófico de la Psicología. Tampoco, y en la misma medida, constituyen un objeto de estudio especial separado e independiente de la conducta, por lo que no existe razón alguna para destacarlos mediante un término propio como si constituyeran una categoría de eventos, de alguna manera, radicalmente distintos de otros procesos conductuales.

\section{Conclusión}

El tratamiento dado a problemas particulares responde siempre a una lógica más básica, por ejemplo, a unas bases ontológicas y epistemológicas definidas. De este modo, cada versión del conductismo postuló visiones diferentes en cuanto al status científico de los eventos privados. Para aquellos adherentes al conductismo metodológico, que se consideraron cercanos al positivismo lógico y al operacionismo, el mundo era reflejado en la experiencia consciente, pero tal experiencia consciente no era en sí misma un objeto de observación ni de análisis, dado que sus contenidos no eran públicamente verificables. El conductismo radical reconoció también la existencia de eventos privados, en la forma de estímulos que solo afectaban a un único individuo, pero propuso que, en tanto estímulos, estos no eran en lo esencial distintos en su naturaleza, de los eventos públicos. La conducta, como un todo, estaba integrada tanto por eventos públicos como privados. Por último, el interconductismo, de una manera aún más radical que el conductismo radical, propuso que los eventos privados son puras construcciones sociales y no, 
como aún sostenía Skinner, eventos internos que existen previamente a la posibilidad de su observación y descripción por el individuo. De este modo, asistimos a un proceso continuo de naturalización de los eventos privados. De eventos inobservables ajenos a toda consideración científica, pasaron a constituir eventos todavía inobservables, pero integrados con los eventos observables en un solo esquema explicativo, para, finalmente, pasar a concebirse como una totalidad en su mayor parte públicamente observable.

\section{Referencias}

Alcaraz, V. M. (1982). La subjetividad como vida interior y como reacción fisiológica. Historia de un concepto. Revista Mexicana de Análisis de la Conducta, 8, 31-37.

Branch, M. N. (2006). How research in behavioral pharmacology informs behavioral science. Journal of the Experimental Analysis of Behavior, 85, 407-423.

Bueno, R. (1993). El conductismo a los ochenta: un análisis tridimensional. Revista Mexicana de Análisis de la Conducta, 19, 97-119.

Bunge, M. (2006). Chasing reality: Strife over realism. Toronto: University of Toronto Press.

Catania, A. C. (1998). Learning (4th ed.). Upper Saddle River, NJ: Prentice Hall.

Clavijo, A. (2007). Lo psicológico como un evento. Universitas Psychologica, 6 (3), 699-711.

Donahoe, J. W. \& Palmer, D. C. (1994). Learning and complex behavior. Boston: Allyn \& Bacon.

Friman, P. C., Wilson, K. G. \& Hayes, S. C. (1998). Behavior analysis of private events is possible, progressive, and nondualistic: A response to Lamal. Journal of Applied Behavior Analysis, 31, 707-708.

Hatfield, G. (2002). Psychology, philosophy, and cognitive science: Reflections on the history and philosophy of experimental psychology. Mind \& Language, 17, 207-232.

Hayes, S. C., Wilson, K. G. \& Guifford, E. V. (1999). Consciousness and private events. En B. A. Thyer (Ed.), The philosophical legacy of behaviorism (pp. 153-187). London: Kluwer Academic.

James, W. (1890). The principles of psychology (Vol. 1). New York: Henry Holt.
Kantor, J. R. (1924-1926). Principles of psychology (Vols. 1-2). New York: Alfred A. Knopf.

Kantor, J. R. (1958). Interbehavioral psychology. Chicago: The Principia Press.

Kantor, J. R. (Pseudonym: Observer, 1981). Concerning the principle of psychological privacy. The Psychological Record, 31, 101-106.

Lashley, K. S. (1923). The behavioristic interpretation of consciousness. Psychological Review, 30, Part 1, 237-272; Part 2, 329-353.

Leahey, T. H. (1992). The mythical revolutions of American Psychology. American Psychologist, 47, 308-318.

Lindsay-Hartz, J., De Rivera, J. \& Mascolo, M. (1995). Differentiating shame and guilt and their effects on motivation. En J. P. Tangney \& K. W. Fischer (Eds.), Self-conscious emotions: Shame, guilt, embarrassment, and pride (pp. 274-300). New York: Guilford Press.

Moore, J. (1985). Some historical and conceptual relations among logical positivism, operationism, and behaviorism. The Behavior Analyst, 8, 53-63.

Ribes, E. (1982). Los eventos privados: iUn problema para la teoría de la conducta? Revista Mexicana de Análisis de la Conducta, 8, 11-29.

Ribes, E. (2004). Behavior is abstraction, not ostension: Conceptual and historical remarks of the nature of Psychology. Behavior and Philosophy, 32, 55-68.

Ribes, E. \& López, F. (1985). Teoría de la conducta: un análisis de campo y paramétrico. México: Trillas.

Ryle, G. (1949). The concept of mind. London: Hutchinson.

Schachter, S. \& Singer, J. E. (1962). Cognitive, social and physiological determinants of emotional state. Psychological Review, 69, 379-399.

Schneider, S. M. \& Morris, E. K. (1987). A history of the term radical behaviorism: From Watson to Skinner. The Behavior Analyst, 10, 27-39.

Skinner, B. F. (1945). The operational analysis of psychological terms. Psychological Review, 52, 270 277; 291-294.

Skinner, B. F. (1953). Science and human behavior. New York: Macmillan.

Skinner, B. F. (1957). Verbal behavior. New York: Appleton-Century-Crofts, Inc. 
Skinner, B. F. (1963). Behaviorism at fifty. Science, 140, 951-958.

Skinner, B. F. (1969). Contingencies of reinforcement: A theoretical analysis. Appleton-Century-Crofts.

Skinner, D. F. (1974). About behaviorism. New York: Alfred A. Knopf.

Skinner, B. F. (1975). The steep and thorny way to a science of behavior. American Psychologist, 30, 42-49.

Smith, L. D. (1986). Behaviorism and logical positivism: A reassessment of the alliance. Stanford, CA: Stanford University Press.

Smith, R. H., Webster, J. M., Parrott, W. G. \& Eyre, H. L. (2002). The role of public exposure in moral and nonmoral shame and guilt. Journal of Personality and Social Psychology, 83, 138-159.

Spence, K. W. (1948). The postulates and methods of behaviorism. Psychological Review, 55, 67-78.

Thomaes, S., Stegge, H. \& Olthof, T. (2010). Does shame bring out the worst in narcissists? En W. Koops, D. Brugman, T. Ferguson \& A. F. Sanders (Eds.), The development and structure of conscience (pp. 221-235). New York: Psychology Press.
Titchener, E. B. (1915). A beginner's psychology. New York: Macmillan.

Titchener, E. B. (1928). A text-book of psychology. New York: Macmillan.

Tolman, E. C. (1927). A behaviorist's definition of consciousness. Psychological Review, 34, 433 439.

Tourinho, E. Z. (2006). Private stimuli, covert responses, and private events: Conceptual remarks. The Behavior Analyst, 29, 13-31.

Uttal, W. R. (2000). The war between mentalism and behaviorism: On the accessibility of the mental processes. Mahwah, NJ: Lawrence Erlbaum.

Watson, J. B. (1913). Psychology as a behaviorist views it. Psychological Review, 20, 158-179.

Watson, J. B. (1914). Behavior: An introduction to comparative psychology. New York: Henry Holt and Company.

Watson, J. B. (1919). Psychology from the standpoint of a behaviorist. Philadelphia: J. B. Lippincot.

Watson, J. B. (1924). Behaviorism. New York: People's Institute Publishing Company. 